\title{
Revisión
}

\section{Guía de la EAU para el Cáncer de Próstata ${ }^{(*)}$}

\author{
Axel Heidenreich, Gunna Aus ${ }^{\mathrm{b}}$, Michel Bollac, Steven Joniau ${ }^{\mathrm{d}}$, Vsevolod B. Matveeve, \\ Hans Peter Schmid ${ }^{\mathrm{f}}$, Filliberto Zattoni ${ }^{\mathrm{g}}$ \\ a Servicio de Urología, Hospital Universitario de Colonia, Colonia, Alemania. \\ ${ }^{b}$ Servicio de Urología, Hospital Universitario de Sahlgrens, Göteborg, Suecia. \\ ${ }^{c}$ Servicio de Radioterapia, C.H.U Grenoble, Grenoble, Francia. \\ ${ }^{d}$ Servicio de Urología, Hospital Universitario de Gasthuisberg, Leuven, Bélgica. \\ e Servicio de Urología, Academia Rusa de Ciencias Médicas, Centro de Investigaciones Oncológicas, Moscú, Rusia. \\ ${ }^{f}$ Servicio de Urología, Kantonspital St. Gallen, St. Gallen, Suiza. \\ ${ }^{g}$ Servicio de Urología, Hospital Santa Maria Della Misericordia, Udine, Italia.
}

Traducción del artículo european uRology 53 (2008) 68-80: Antonio Rosino SáncheZ

Servicio de Urología. Hospital Morales Meseguer, Murcia, España.

\section{Resumen}

Objetivos: Presentar un resumen de la versión del 2007 de la guía de la Asociación Europea de Urología (EAU) para el cáncer de próstata (CaP).

Métodos: Se realizó por un grupo de trabajo una revisión de los nuevos datos presentes desde 2004 hasta 2007 en la literatura. Las guías han sido actualizadas y el nivel de evidencia/grado de recomendación ha sido añadido al texto basándose en una revisión sistemática de la literatura, que incluía una búsqueda de las bases de datos online y revisiones bibliográficas.

Resultados: Una versión completa está disponible en la EAU Office o en www.uroweb.org. El método diagnóstico de elección es la biopsia sistematizada de la próstata bajo control ecográfico. El tratamiento activo es el recomendado en la mayoría de los pacientes con enfermedad localizada y una larga esperanza de vida, siendo la prostatectomía radical superior a la vigilancia ("watchful waiting") en un ensayo randomizado prospectivo. La prostatectomía radical con preservación de bandeletas es la técnica de elección en la enfermedad órgano-confinada; el bloqueo androgénico neoadyuvante no ha demostrado una mejoría en las variables de resultados. La radioterapia debe realizarse con al menos 72 Gy en el CaP de bajo riesgo y con 78 Gy en el de intermedio - alto riesgo. El bloqueo androgénico en monoterapia es el estándar del tratamiento en el CaP metastásico; el bloqueo androgénico intermitente podría ser un tratamiento alternativo en pacientes seleccionados. El seguimiento se basa principalmente en los niveles de PSA y en la anamnesis específica de la enfermedad, estando las pruebas de imagen sólo indicadas cuando aparecen los sintomas. La quimioterapia con docetaxel ha surgido como el tratamiento de referencia para el CaP metastásico hormonorefractario.

Conclusiones: El conocimiento en el campo del CaP está rápidamente cambiando. Estas guías de la EAU resumen los hallazgos más recientes y los aplican a la práctica clínica.

Palabras clave: Bloqueo hormonal. Quimioterapia. Diagnóstico. Guías de la EAU. Seguimiento. Cáncer de próstata. Radioterapia. Prostatectomía radical. Revisión. Tratamiento.

\section{EAU Guidelines on Prostate Cancer}

\section{Abstract}

Objectives: To present a summary of the 2007 version of the European Association of Urology (EAU) guidelines on prostate cancer (PCa). Methods: A literature review of the new data emerging from 2004 to 2007 was

performed by the working panel. The guidelines have been updated, and the level of evidence/grade of recommendation was added to the text based on a systematic review of the literature, which included a search of online databases and bibliographic reviews.

Results: Afull version is available at the EAU Office or at www.uroweb.org. Systemic prostate biopsy under ultrasound guidance is the preferred diagnostic method.

Active treatment is mostly recommended for patients with localized disease and a long life expectancy, with radical prostatectomy being shown to be superior to watchful waiting in a prospective randomized trial. Nerve-sparing radical prostatectomy represents the approach of choice in organ-confined disease; neoadjuvant androgen deprivation demonstrates no improvement of outcome variables. Radiation therapy should be performed with at least 72 and 78 Gy in low-risk and intermediate- to high-risk PCa, respectively. Monotherapeutic androgen deprivation is the standard of care in metastatic PCa; intermittent androgen deprivation might be an alternative treatment option for selected patients. Follow-up is largely based on prostate-specific antigen and a disease-specific history with imaging only indicated when symptoms occur. Cytotoxic therapy with docetaxel has emerged as the reference treatment for metastatic hormone-refractory PCa.

Conclusions: The knowledge in the field of PCa is rapidly changing. These EAU guidelines on PCa summarize the most recent findings and put them into clinical practice.

Keywords: Androgen deprivation. Chemotherapy. Diagnosis. EAU guidelines. Follow-up. Prostate cancer. Radiation therapy. Radical prostatectomy. Review. Treatment.

(*)La traducción de este artículo se ha llevado a cabo con el permiso de la Asociación Europea de Urología. 


\section{Introducción}

El último resumen de las guías para el cáncer de próstata (CaP) de la Asociación Europea de Urología (EAU) se publicó en el $2005^{1}$. La versión larga de estas guías está continuamente actualizándose debido a los muy importantes cambios que se han producido en los últimos años que afectan al manejo clínico del CaP. El propósito de este trabajo es presentar un resumen de la actualización del 2007 de la guía de la EAU para el CaP. Para facilitar la evaluación de la calidad de la información suministrada, los grados de evidencia y el grado de recomendación han sido incluidos de acuerdo con los principios generales de la medicina basada en la evidencia ${ }^{2}$.

\section{Epidemiologia}

Se admite que el CaP es uno de los principales problemas de salud que debe afrontar la población masculina. En Europa, se estima que se diagnostican 2,6 millones de nuevos casos cada año. El CaP constituye aproximadamente el $11 \%$ de todas las neoplasias en los varones de Europa ${ }^{3}$, y es el responsable del 9\% de las muertes por cáncer entre los hombres dentro de la Unión Europea ${ }^{4}$.

\section{Factores de riesgo}

Los factores hereditarios son importantes en la determinación del riesgo de desarrollar un CaP clínico, y los factores exógenos pueden tener una influencia decisiva sobre esta predisposición. La pregunta clave es si hay suficientes datos para recomendar cambios en el estilo de vida para disminuir ese riesgo. Hay algunos datos al respecto y esta información se podría dar a los parientes varones de pacientes con CaP que preguntasen por la influencia de la dieta (nivel de evidencia: 3-4).

Si un familiar de primer grado tiene la enfermedad, el riesgo por lo menos se dobla. Si dos o más familiares de primer grado están afectados, el riesgo se incrementa de 5 a 11 veces $^{5}$. Una pequeña subpoblación de pacientes con CaP (alrededor del 9\%) tiene un verdadero CaP hereditario, definido como tres o más familiares afectados o dos parientes que hayan desarrollado precozmente la enfermedad, es decir, antes de los 55 años de edad ${ }^{6}$.

\section{Clasificaciones}

La clasificación tumor, ganglios (“nodes”), metástasis (TNM) del año 2002 de la Unión Internacional Contra el Cáncer es la utilizada a lo largo de estas guías ${ }^{7}$. El sistema más comúnmente utilizado para clasificar el adenocarcinoma de próstata es el grado Gleason $^{8}$. Este sistema da una puntuación entre 2 y 10 , siendo el grado 2 el menos agresivo y el 10 el más agresivo. Esta puntuación es la suma de los dos patrones más frecuentes (grado 1-5) encontrados en el tumor. Para ser tenido en cuenta, un patrón (grado) necesita ocupar más del 5\% del espécimen de la biopsia.

\section{Cribaje del cáncer de próstata}

El cribaje poblacional o de masas se define como un examen sistemático de hombres asintomáticos (en riesgo). Normalmente el cribaje se engloba dentro del marco de un ensayo o estudio y es iniciado por la organización o estructura que realiza el estudio. Por el contrario, el cribaje oportunista busca la detección precoz del CaP en casos individuales y es iniciado por el paciente o por su médico. Dos grandes estudios aleatorizados están en marcha para evaluar la eficacia del cribaje del CaP: el "Prostate, Lung, Colorectal and Ovary Trial” (Estudio para Próstata, Pulmón, Colorrectal y Ovario) en los EEUU y el "European Randomized Screening for Prostate Cancer" (Cribaje Aleatorizado Europeo para Cáncer de Próstata) ${ }^{9}$. Los primeros análisis de las principales variables de resultados de estos ensayos - diferencias en mortalidad por CaP están previstas para el 2013 y el 2008 respectivamente (nivel de evidencia 1b).

Por tanto, en estos momentos, carecemos de datos para apoyar o desaconsejar la instauración sistemática de programas de cribaje poblacional para la detección precoz del cáncer de próstata orientado a todos los hombres de una determinada población (nivel de evidencia: 3). El uso del antígeno prostático específico (PSA) en combinación con el tacto rectal como una ayuda para el diagnóstico precoz en pacientes bien informados, es menos controvertido y ampliamente usado en la práctica clínica $^{10,11}$ (Nivel de evidencia: 3 ).

\section{Diagnóstico y estadiaje del cáncer de próstata}

Las herramientas diagnósticas principales utilizadas para buscar datos de la existencia de un CaP son el tacto rectal, la concentración sérica de PSA, y las biopsias prostáticas transrectales ecodirigidas ${ }^{11}$. El diagnóstico depende de la presencia de adenocarcinoma en las muestras quirúrgicas o en los cilindros de las biopsias prostáticas. 
El punto de corte de PSA que indique el mayor riesgo de $\mathrm{CaP}$ necesita ser definido ${ }^{10}$. Extrapolando los datos de las curvas, el riesgo acumulado a los 7 años de ser diagnosticado de un $\mathrm{CaP}$ en un programa de cribaje basado en el PSA fue sólo del 34\% para los hombres con PSA entre 3 y $6 \mathrm{ng} / \mathrm{ml}, 44 \%$ para aquéllos con valores de PSA entre 6 y $10 \mathrm{ng} / \mathrm{ml}$ y del $71 \%$ para los varones con niveles de PSA $>10$ $\mathrm{ng} / \mathrm{ml}^{12}$. En hombres más jóvenes (50 - 66 años), la tasa de detección de CaP fue del 13,2\% en el rango de PSA entre 3-4 $\mathrm{ng} / \mathrm{ml}^{12}$. El hallazgo de que muchos hombres podrían esconder un $\mathrm{CaP}$ a pesar de valores bajos de PSA ha sido subrayado por los resultados recientes de un estudio de prevención realizado en los EE.UU ${ }^{13}$.

En la práctica clínica rutinaria, se acepta que un ratio PSA libre/PSA total $<20 \%$ y una velocidad de PSA $>0,75 \mathrm{ng} / \mathrm{ml} /$ año en hombres con niveles de PSA elevados, son parámetros válidos que se asocian a un mayor riesgo de $\mathrm{CaP}$ y que facilitan la indicación de la realización de una biopsia prostática. En un estudio retrospectivo reciente sobre 12.078 pacientes sometidos a una biopsia de próstata $^{14}$, se identificaron los valores de corte de PSA y de velocidad de PSA útiles para mejorar la valoración del riesgo de CaP en hombres $<50$ años. La prevalencia de $\mathrm{CaP}$ fue del $4,4 \%$ y $14,2 \%$ en varones $<50$ y $\geq 50$ años respectivamente. Aunque el estudio podría estar sesgado por la asignación o por la valoración, un punto de corte de PSA de $2,5 \mathrm{ng} / \mathrm{ml}$ y una velocidad de PSA $>0,60 \mathrm{ng} / \mathrm{ml} /$ año parecen ser los adecuados para la práctica clínica.

Las biopsias transrectales ecoguiadas dirigidas lateralmente de un calibre $18 \mathrm{G}$ se han convertido en el estándar en la obtención de material para el examen anatomopatológico. En número de biopsias que son precisas para una óptima detección del $\mathrm{CaP}$ está todavía en discusión. Prácticamente todos los estudios han mostrado una mayor detección de cáncer al aumentar el número de muestras en comparación con la técnica sextante estándar ${ }^{15}$. Actualmente, se recomienda la toma de al menos 10 cilindros o el uso de los nomogramas de Viena como técnica rutinaria; las variaciones se deben realizar teniendo en cuenta la edad, los niveles de PSA y el volumen prostático. Los estudios muestran claramente que la zona transicional no debería ser un área objetivo en la primera tanda de biopsias por la constante baja tasa de detección $\leq 2 \%{ }^{16}$. Si la primera tanda de biopsias es negativa, se puede recomen- dar repetir la biopsia. Se ha informado de una tasa de detección de alrededor del 10-35\% en segundas biopsias tras una primera biopsia negativa ${ }^{17}$. En los casos en los que haya neoplasia intraepitelial prostática de alto grado (PIN alto grado o HGPIN) o proliferación atípica de pequeño acino (ASAP), aproximadamente el $30-50 \%$ de las próstatas presentan un cáncer concomitante y una nueva biopsia debe ser indicada.

La decisión de proseguir con nuevas pruebas diagnósticas o de estadiaje dependen de qué opciones terapéuticas están disponibles para el enfermo, teniendo en cuenta sus preferencias, edad y comorbilidades. Se puede generalmente prescindir de aquellos procedimientos que no vayan a afectar a la decisión terapéutica. Un breve resumen de las guías para el diagnóstico y estadiaje se presenta en la Tabla 1.

\section{Tratamiento primario local del cáncer de próstata}

No es posible afirmar que un tratamiento es claramente mejor que otro, ya que carecemos de ensayos clínicos aleatorizados al respecto. Sin embargo, basándose en la literatura disponible, se pueden hacer algunas recomendaciones. Un resumen, dividido por estadío al diagnóstico, se presenta en la Tabla 2; más abajo se hacen unas pocas recomendaciones en referencia a las diferentes opciones terapéuticas. En general, se recomienda integrar los nomogramas desarrollados y validados recientemente en el proceso de decisión.

\subsection{Vigilancia activa}

Los términos "tratamiento diferido" o "watchful waiting" (esperar y vigilar) se usan para describir una estrategia de tratamiento que incluye una política activa de posponer el tratamiento hasta que éste sea preciso. Hay que seguir de forma estrecha a los pacientes a los que se les ofrece "watchful waiting". La vigilancia activa ha surgido como una alternativa terapéutica en hombres con CaP y bajo riesgo de progresión de la enfermedad basándose en los datos iniciales de Chodak et al. ${ }^{18}$ y Albertsen et al. ${ }^{19}$ que demostraban que los varones con $\mathrm{CaP}$ bien diferenciados tienen una supervivencia cáncer específica a los 20 años del 80-90\%. El grado Gleason, el estadío clínico y el PSA son factores de riesgo ampliamente aceptados que predicen la probabilidad de progresión. 
Tabla 1. Guía en el diagnóstico y estadiaje del cáncer de próstata

1. Un tacto rectal anormal o una elevación en los niveles del PSA sérico pueden indicar un CaP. El punto de corte exacto de lo que se debe considerar un PSA normal todavía no se ha determinado, pero valores alrededor $<2$ '5- $3 \mathrm{ng} / \mathrm{ml} \mathrm{son}$ los usados habitualmente para los hombres más jóvenes (grado C de recomendación)

2. El diagnóstico de $\mathrm{CaP}$ depende de una confirmación histopatológica (o citológica) (grado B de recomendación). La biopsia y el estadiaje posterior sólo están indicados si van a modificar el manejo del paciente (grado C de recomendación).

3. En la mayoría de los casos con sospecha de CaP, el método diagnóstico recomendado es la biopsia sistemática transrectal ecodirigida. Se recomienda tomar un mínimo de 10 cilindros dirigidos lateralmente o el uso del nomograma de Viena, con más cilindros en las glándulas más grandes (grado B de recomendación). No se recomiendan las biopsias de la zona transicional en la primera tanda de biopsias dada su baja tasa de detección (grado C de recomendación).

- Está justificado repetir una tanda de biopsias en aquellos casos con persistencia en la indicación (tacto rectal anormal, PSA elevado, o hallazgos histopatológicos sugerentes de malignidad en la primera biopsia) de biopsia prostática (grado B de recomendación).

- No se pueden hacer recomendaciones globales para subsiguientes (tercera o posteriores) tandas de biopsias prostáticas; la decisión tiene que ser tomada en base a cada paciente en particular (grado C de recomendación).

4. Se puede ofrecer a los pacientes la inyección periprostática transrectal con un anestésico local como una analgesia efectiva para la realización de biopsias prostáticas (grado A de recomendación).

5. El estadiaje local (estadío T) del CaP se basa en los hallazgos del tacto rectal y, posiblemente, de las imágenes de la resonancia magnética. Se puede obtener información adicional del número y sitio de las biopsias positivas prostáticas, el porcentaje de afectación del cilindro, el grado tumoral, y los niveles de PSA sérico (grado C de recomendación).

6. La afectación linfática (estadío N) es sólo importante cuando se plantea un tratamiento potencialmente curativo. Los pacientes con $\mathrm{CaP}$ de riesgo moderado o alto tienen una probabilidad $>10 \%$ de presentar metástasis linfáticas y debieran someterse a una linfadenectomía pélvica. Un estadiaje linfático adecuado sólo se puede conseguir mediante una linfadenectomía ampliada (grado B de recomendación).

7. La mejor forma de valorar las metástasis esqueléticas (estadiaje M) es la gammagrafía ósea. No se debe indicar en pacientes asintomáticos con un tumor bien o moderadamente diferenciado, si los niveles de PSA sérico son $<20 \mathrm{ng} / \mathrm{ml}$ (grado $\mathrm{B}$ de recomendación).

De acuerdo con datos recientes, los hombres con $\mathrm{CaP}$ de bajo riesgo (PSA $<10 \mathrm{ng} / \mathrm{ml}$, grado Gleason $\leq 6$, cT1c - cT2a, esperanza de vida <10 años) son buenos candidatos para la vigilancia activa ${ }^{20}$. Los varones con una esperanza de vida >15 años podrían ser candidatos si están afectados menos de tres cilindros con $<50 \%$ de afectación en cada uno de los cilindros. Una reciente actualización de 299 pacientes con una mediana de seguimiento de 64 meses, mostró que el 34\% de los pacientes abandonaban la vigilancia activa debido a progresión bioquímica, clínica o histológica, o por preferencia del paciente ${ }^{21}$. A los 8 años, la supervivencia global es del 85\% y la supervivencia cáncer específica del 99\%. Un tiempo de doblaje de PSA >3 años y la biopsia de repetición parecen ser herramientas útiles para valorar un tratamiento activo en los pacientes de bajo riesgo diagnosticados mediante cribaje y manejados inicialmente de manera expectante.

\subsection{Prostatectomía radical}

De momento, la prostatectomía radical (PR) es el único tratamiento para el CaP localizado que ha demostrado un beneficio en la supervivencia cáncer específica comparada al manejo conservador en un ensayo prospectivo y aleatorizado ${ }^{22}$. La PR con preservación de bandeletas representa la técnica de elección en todos los hombres con erecciones normales y enfermedad organoconfinada, mejorando la calidad de vida sin comprometer los resultados oncológicos ${ }^{23}$. La necesidad de una linfadenectomía ampliada es, de momento, controvertida. Sin embargo, la probabilidad de afectación de los ganglios linfáticos es baja en los hombres con CaP de bajo riesgo (cT1c, PSA $<10 \mathrm{ng} / \mathrm{ml}$, grado Gleason en la biopsia $\leq 6$ ) con $<50 \%$ de los cilindros positivos. En los varones de riesgo intermedio (cT2a, PSA 10$20 \mathrm{ng} / \mathrm{ml}$, grado Gleason de la biopsia =7) o alto (cT2b, PSA >20 ng/ml, grado Gleason de la biopsia $\geq 8$ ) debería realizarse siempre una linfadenectomía ampliada debido al relativamente alto riesgo de afectación de los ganglios linfáticos ${ }^{24}$.

El manejo primario de los CaP estadio cT3 tiene que ser multimodal debido a la alta probabilidad de afectación de los ganglios linfáticos y/o márgenes positivos de la resección. El sobrestadiaje del CaP cT3 es relativamente frecuente y ocurre en el 13$27 \%$ de los casos. Aunque todavía está en discusión, es cada vez más evidente que la cirugía tiene un lugar en el tratamiento de la enfermedad localmente 
Tabla 2. Guía del tratamiento primario del cáncer de próstata

\begin{tabular}{|c|c|c|}
\hline Estadio & Tratamiento & Comentario \\
\hline \multirow[t]{5}{*}{ Tla } & Esperar y vigilar (WW) & $\begin{array}{l}\text { Tratamiento estándar para el CaP con puntuación Gleason } \leq 7 \text { y }<10 \text { años de esperanza } \\
\text { de vida. En pacientes con }>10 \text { años de esperanza de vida, se recomienda reestadiar con } \\
\text { ETR y biopsia (grado B de recomendación). }\end{array}$ \\
\hline & Prostatectomía radical & $\begin{array}{l}\text { Opcional en pacientes jóvenes con una larga esperanza de vida, especialmente si el grado } \\
\text { Gleason es 8-10 (grado B de recomendación). }\end{array}$ \\
\hline & Radioterapia & $\begin{array}{l}\text { Opcional en pacientes jóvenes con una larga esperanza de vida, especialmente si el } \\
\text { grado Gleason es 8-10. Mayor riesgo de complicaciones tras RTU-P, especialmente con la } \\
\text { radioterapia intersticial (grado B de recomendación). }\end{array}$ \\
\hline & Hormonoterapia & No es una opción (grado A de recomendación). \\
\hline & Combinación & No es una opción (grado C de recomendación). \\
\hline
\end{tabular}

T1b-T2b Esperar y vigilar (WW)

Pacientes asintomáticos con grado Gleason en la biopsia $\leq 7$ y $<10$ años de esperanza de vida. Pacientes que no acepten las complicaciones asociadas a los tratamientos curativos (grado B de recomendación).

Prostatectomía radical Tratamiento estándar para los pacientes con una esperanza de vida >10 años que acepten las complicaiones asociadas al tratamiento (grado A de recomendación).

Radioterapia

Pacientes con una esperanza de vida $>10$ años que acepten las complicaciones asociadas al tratamiento. Pacientes con contraindicación para la cirugía. No adecuada para pacientes con una esperanza de vida de 5-10 años y un grado Gleason 8-10 (se recomienda tratamiento combinado; ver debajo) (grado B de recomendación).

Hormonoterapia Pacientes sintomáticos que precisan una paliación de sus síntomas y no son aptos para un tratamiento curativo (grado C de recomendación).

Los antiandrógenos puros se asocian a peores resultados en comparación con la WW o vigilancia, y no se recomiendan (grado A de recomendación).

Combinación

HTN + prostatectomía radical: No se ha demostrado ningún beneficio (grado A de recomendación).

HTN + radioterapia: mejor control local. No se ha demostrado un beneficio en supervivencia (grado $\mathrm{B}$ de recomendación).

Hormonoterapia (2-3 años) + radioterapia: mejor que radioterapia sola en tumores poco diferenciados (grado A de recomendación).

T3-T4 Esperar y vigilar (WW)

Prostatectomía radical

Es una opción en pacientes asintomáticos con estadío T3, grado Gleason $\leq 7$, y una esperanza de vida $<10$ años (grado $\mathrm{C}$ de recomendación).

Opcional para pacientes seleccionados con una enfermedad limitada $\leq \mathrm{T} 3 \mathrm{a}$, Gleason $\leq 8$, PSA $<20 \mathrm{ng} / \mathrm{ml}$, y una esperanza de vida $>10$ años (grado C de recomendación).

Radioterapia T3 con una esperanza de vida >5-10 años. Parece ser beneficiosa una escalada de dosis $>70$ Gy. Si esto no está disponible, se podría recomendar una combinación con tratamiento hormonal (ver debajo) (grado A de recomendación).

Hormonoterapia

Pacientes sintomáticos, extensa enfermedad T3-T4, niveles de PSA elevados (>25 ng/ml), pacientes con mal estado de salud. Mejor que vigilancia (WW) (grado A de recomendación). Radioterapia + tratamiento hormonal parece ser mejor que la radioterapia sola (grado A de recomendación).

Combinación

HTN + prostatectomía radical: no se ha demostrado ningún beneficio (grado B de recomendación).

$\mathrm{N}+$, Mo $\quad$ Esperar y vigilar (WW):

Pacientes asintomáticos. Solicitado por el paciente. Puede haber una influencia negativa sobre la supervivencia (grado $\mathrm{C}$ de recomendación).

No es una opción estándar (grado C de recomendación).

Prostatectomía radical Radioterapia

Hormonoterapia

Combinación

No es una opción estándar (grado C de recomendación).

Tratamiento estándar (grado A de recomendación).

No es un tratamiento estándar. Solicitado por el paciente (grado B de recomendación).

$\mathrm{M}+\quad$ Esperar y vigilar (WW)

No es un tratamiento estándar. Puede causar una peor supervivencia/más complicaciones que con el tratamiento hormonal inmediato (grado B de recomendación).

Prostatectomía radical

Radioterapia

Hormonoterapia

No es una opción (grado $\mathrm{C}$ de recomendación).

No es una opción (como tratamiento curativo) (grado C de recomendación).

Tratamiento estándar. No se debe negar este tratamiento a los pacientes sintomáticos (grado A de recomendación).

Combinación

No es una opción (grado C de recomendación).

Hormonoterapia: todas las formas de tratamiento hormonal; Combinación: hormonoterapia previa y/o tras prostatectomía radical o radioterapia; wW: watchful waiting; ETR: ecografía transrectal; RTU-P: resección transuretral de próstata; HTN: hormonoterapia neoadyuvante. Para información más detallada y discusión acerca de tratamientos de segunda línea, por favor consulten la versión completa de las guías. 
avanzada con excelentes supervivencias globales y cáncer específicas a los 5, 10 y 15 años de 95\%, $90 \%$ y $79 \%$ respectivamente ${ }^{25,26}$.

El bloqueo androgénico neoadyuvante antes de la PR no ofrece una ventaja significativa en la supervivencia global ni en la supervivencia libre de progresión según un reciente meta-análisis en Cochrane $^{27}$. Aún más, no se asocia con una mejoría de las variables patológicas locales.

El bloqueo androgénico adyuvante tras una PR siempre ha sido controvertido ${ }^{28}$. Aunque el único ensayo prospectivo y aleatorizado demostró una mejoría significativa en la supervivencia con el bloqueo androgénico inmediato en la enfermedad $\mathrm{N}+{ }^{29}$, se debe recordar que la mayoría de los pacientes tenían adenopatías macroscópicas y que el 70\% tenían también márgenes positivos y/o afectación de las vesículas seminales. No está claro si el bloqueo androgénico adyuvante en pacientes con afectación mínima linfática tendría los mismos resultados beneficiosos. La actualización más reciente del "Early Prostate Cancer Trial” (Ensayo del Cáncer de Próstata Precoz), ha mostrado que no hay ningún beneficio en la supervivencia libre de progresión añadiendo 150 $\mathrm{mg} /$ día de bicalutamida al manejo habitual, mientras que se identificó un beneficio en la supervivencia global de los varones con CaP localmente avanzado ${ }^{28}$. No se pueden hacer recomendaciones finales para los pacientes con afectación microscópica de los ganglios linfáticos exclusivamente.

\subsection{Radioterapia}

En la radioterapia externa, se recomienda al menos una dosis de 72 Gy en el manejo de los CaP de bajo riesgo, ya que se ha demostrado que la supervivencia libre de enfermedad bioquímica es significativamente más alta con dosis de radiación $\geq 72$ Gy comparados con $<72$ Gy (69\% vs. 63\%, p=0,046) . $^{30}$.

Para el CaP de riesgo intermedio, muchas series han demostrado un impacto significativo de la escalada de dosis en la supervivencia libre de recidiva bioquímica a los 5 años en los pacientes clasificados como cT1c-cT3, con un rango de dosis de 76 a 81 $\mathrm{Gy}^{31}$. En la práctica diaria, aunque no se ha alcanzado un consenso acerca de la magnitud del incremento de la dosis, 78 Gy aparentemente es un buen acuerdo. Para los pacientes que reciben hasta $72 \mathrm{~Gy}$, una pauta corta de 6 meses de bloqueo androgénico es beneficioso, pero no está claro su papel en el tratamiento con altas dosis de radiación.
En pacientes con enfermedad de alto riesgo, la radioterapia externa con escalada de dosis mejora la supervivencia libre de enfermedad bioquímica ${ }^{32}$, pero parece ser insuficiente para prevenir el riesgo de recidiva fuera de las pelvis. Por tanto, es obligatorio el bloqueo androgénico neoadyuvante y adyuvante durante 2 años para mejorar de forma significativa la supervivencia global y cáncer específica ${ }^{33,34}$.

\subsection{Braquiterapia transperineal}

La braquiterapia transperineal de baja dosis es una técnica segura y eficiente que puede ser aplicada a los pacientes con los siguientes criterios de selección: estadío cT1b-T2a NO MO, grado Gleason $\leq 6$ valorado sobre un número suficiente de biopsias aleatorizadas, un PSA inicial $\leq 10 \mathrm{ng} / \mathrm{ml}, \leq 50 \%$ de los cilindros de la biopsia afectados con cáncer, un volumen prostático $<50 \mathrm{~cm}^{3}$ y una buena puntuación en el IPSS (International Prostatic Symptom Score $)^{35}$. Distintos centros han informado de los resultados con implantes permanentes con una mediana de seguimiento que oscila entre 36 y 120 meses $^{36}$. Se ha informado una supervivencia libre de recidiva tras 5 y 10 años que varía entre $71 \%$ y $93 \%$ y entre $65 \%$ a $85 \%$ respectivamente. El añadir un bloqueo androgénico neoadyuvante o adyuvante a la braquiterapia transperineal de baja dosis no ha mostrado ningún beneficio ${ }^{36}$. La mayoría de los pacientes presentan síntomas urinarios agudos al poco tiempo tras la colocación de los implantes, tales como retención urinaria $(1,5-22 \%)$ con la consiguiente posterior RTU prostática (hasta un 8,7\%) e incontinencia (0-19\%). Hasta un 20\% de los pacientes pueden presentar morbilidad crónica urinaria, dependiendo de la gravedad de los síntomas previos a la braquiterapia.

\subsection{Radioterapia externa adyuvante en los}

cánceres de próstata pT3 o pTxR1

Tres ensayos prospectivos aleatorizados han valorado el papel de la radioterapia postoperatoria inmediata $^{37}$. El estudio EORTC 22911 comparaba la radioterapia inmediata postoperatoria (60 Gy) a la radioterapia diferida hasta la presencia de una recidiva local (70 Gy) en pacientes clasificados como pT3 pNO tras una PR retropúbica. La radioterapia inmediata postoperatoria demostró ser bien tolerada, con un riesgo de toxicidad urinaria grado 3-4 menor del 3,5\%. Todos los ensayos concluyen que la radioterapia postoperatoria inmediata tras la cirugía 
mejora significativamente la supervivencia libre de recidiva clínica o biológica a los 5 años en aproximadamente un 20\%. Sin embargo, no se ha demostrado que la radioterapia inmediata mejora la supervivencia libre de metástasis ni la supervivencia cáncer específica en esta cohorte de pacientes. Los candidatos más adecuados para la radioterapia inmediata podrían ser aquéllos con márgenes positivos multifocales, un grado Gleason $<7$, o PSA nadir $\geq 0,1 \mathrm{ng} / \mathrm{ml}$ tras la PR.

\section{Opciones terapéuticas alternativas del cáncer de próstata localizado}

Aparte de la PR, la radioterapia externa y/o la braquiterapia, han surgido como opciones terapéuticas la crioterapia de la próstata y el HIFU ("high intensity focused ultrasound") para aquellos pacientes con CaP clínicamente localizado que no son adecuados para una $\mathrm{PR}^{38}$. Los pacientes candidatos ideales para la crioablación de la próstata son aquellos con enfermedad organoconfinada, tamaño prostático $\leq 40 \mathrm{ml}$, PSA sérico < $20 \mathrm{ng} / \mathrm{ml}$, y un grado Gleason en la biopsia $<7$. Dado que hay sólo pocos datos acerca de los resultados a largo plazo en términos de control del cáncer, los pacientes con una esperanza de vida $>10$ años deberían ser informados al respecto.

\section{Terapia hormonal}

Hoy en día, los agonistas de la hormona liberadora de hormona luteinizante (LHRH) se han convertido en el tratamiento estándar dentro de la terapia hormonal, ya que evitan las secuelas físicas y psicológicas asociadas a la orquiectomía y carecen de la cardiotoxicidad potencial asociada al $\mathrm{DES}^{39,40}$. Como tratamiento primario antiandrógeno en monoterapia, se ha comparado $150 \mathrm{mg} /$ día de bicalutamida con la castración médica o quirúrgica en dos grandes estudios prospectivos aleatorizados con un idéntico diseño de estudio, incluyendo un total de 1435 pacientes con CaP localmente avanzado M0 o M1 ${ }^{41}$ En los pacientes M1, se demostró una mejoría en la supervivencia global en el grupo de castración, aunque la diferencia en la mediana de supervivencia entre ambos grupos fue de sólo 6 semanas. Los antiandrógenos no esteroideos podrían ser una alternativa terapéutica en un subgrupo de pacientes M1 si el PSA sérico es $\leq 400 \mathrm{ng} / \mathrm{ml}$, mientras que los antiandrógenos esteroideos no lo son ${ }^{42}$. En los pacientes MO, no se halló una diferencia significativa en la supervivencia global. Las indicaciones actuales para el bloqueo androgénico se resumen en la Tabla 3 .

Tabla 3. Indicaciones de la hormonoterapia

\begin{tabular}{ll}
\hline Indicación dela castración & Comentarios \\
\hline M1 sintomática & $\begin{array}{l}\text { Para paliar los sintomas y reducir el riesgo de secuelas potencialmente catastróficas } \\
\text { de la enfermedad avanzada (compresión de la médula espinal, fracturas patológicas, } \\
\text { obstrucción ureteral, metástasis extraesqueléticas) (nivel de evidencia: } 3 \text { ) }\end{array}$
\end{tabular}

M1 asintomática

$\mathrm{N}+$

Localmente avanzado MO

Localmente avanzado sintomático

Localmente avanzado asintomático

no apto para tratamiento local definitivo.

Antiandrógenos

Administración a corto plazo:

Antiandrógenos no esteroideos:
Castración inmediata para retrasar la progresión a un estadío sintomático y prevenir complicaciones importantes relacionadas con la progresión de la enfermedad (nivel de evidencia: $1 b)$

Castración inmediata en metástasis macroscópicas para prolongar la supervivencia libre de progresión (nivel de evidencia: 1b) e incluso supervivencia global (nivel de evidencia: 3). No se puede hacer una recomendación final para afectación ganglionar microscópica.

Castración inmediata para mejorar la supervivencia global (nivel de evidencia: 1b)

Nivel de evidencia: 4

Para reducir el riesgo del fenómeno llamarada en pacientes con enfermedad metastásica avanzada que van a recibir agonistas de hormona liberadora de hormona luteinizante (agonistas LHRH) (nivel de evidencia: 1b)

Monoterapia primaria como alternativa a la castración en pacientes con CaP localmente avanzado (nivel de evidencia: 1b) 


\subsection{Bloqueo androgénico completo}

Las más recientes revisiones sistemáticas y meta-análisis dan, a los 5 años de seguimiento, una pequeña ventaja de supervivencia $(<5 \%)$ al bloqueo androgénico completo comparado con la monoterapia ${ }^{39,41}$. Sigue siendo cuestionable si esta pequeño ventaja, si real, puede ser de alguna significación cuando se aplica a la práctica clínica diaria.

\subsection{Bloqueo androgénico intermitente}

La idea del bloqueo androgénico intermitente es la de preservar la calidad de vida y reducir los costes asociados al tratamiento sin comprometer la eficacia terapéutica de la manipulación hormonal. Varios estudios en fase 2 han demostrado la viabilidad del bloqueo androgénico intermitente en la enfermedad metastásica o en la recurrencia bioquímica, con una tasa de respuesta del PSA y de mejoría de los síntomas similar al bloqueo androgénico completo. El trabajo más importante SWOG 9346) aleatorizó a 1.134 hombres con CaP estadío D2 a bloqueo androgénico completo o intermitente tras 7 meses de inducción de bloqueo y una reducción de $\mathrm{PSA}<4 \mathrm{ng} / \mathrm{ml}^{43}$. La reducción del PSA a $<0,2,<4$ y $>4 \mathrm{ng} / \mathrm{ml}$ fue identificado como un factor pronóstico significativo en relación con la supervivencia, siendo de 75, 44 y 13 meses respectivamente. Por tanto, se puede ofrecer bloqueo androgénico intermitente a pacientes seleccionados pero aún carecemos de resultados de estudios aleatorizados. Parece ser preciso un periodo mínimo de inducción de 7 meses con tratamiento hormonal continuo y una reducción del PSA a $<4 \mathrm{ng} / \mathrm{ml}$ para que el protocolo intermitente tenga éxito.

\subsection{Bloqueo androgénico inmediato vs. diferido}

El momento más adecuado para introducir el tratamiento hormonal en pacientes con CaP avanzado está todavía en discusión. No está claro si el bloqueo inmediato en la enfermedad localmente avanzada o metastásica asintomática tiene una influencia positiva en la supervivencia y en la calidad de vida comparado con el bloqueo diferido hasta el momento en que haya sintomas. La duda se deriva de la falta de ensayos controlados aleatorizados bien dirigidos.

En relación con la elevación del PSA tras una PR, también carecemos de estudios aleatorizados prospectivos. Sólo disponemos de un análisis retrospectivo de 1352 varones con elevación del PSA tras
$\mathrm{PR}^{44}$. De estos 1.353 hombres, 355 comenzaron con niveles de PSA diversos el bloqueo androgénico mientras que 997 estuvieron sin manipulaciones hormonales hasta la detección de enfermedad metastásica. El bloqueo androgénico precoz mostró un beneficio en relación con el intervalo libre de metástasis óseas sólo en los pacientes con un grado Gleason > 7 o un PSA-DT < 12 meses; no se halló una diferencia estadísticamente significativa en la supervivencia global o cáncer específica.

\section{Seguimiento de los pacientes con cáncer de próstata}

Los pacientes diagnosticados de $\mathrm{CaP}$ se siguen habitualmente de por vida o hasta una edad tan avanzada que haga el seguimiento superfluo. Las pruebas básicas en el seguimiento de los pacientes con CaP son la determinación de los niveles séricos de PSA, la anamnesis específica y el tacto rectal. No se recomienda realizar de rutina pruebas de imagen y éstas deben ser reservadas para situaciones específicas. No se ha estudiado bien la periodicidad de las revisiones y qué pruebas son necesarias en cada visita, y frecuentemente deben ser individualizadas. La Tabla 4 resume las guías para el seguimiento tras un tratamiento con intención curativa y en la Tabla 5 están las guías para el seguimiento con bloqueo androgénico. Los pacientes manejados inicialmente con vigilancia activa necesitan un seguimiento personalizado dependiendo de cuáles son los objetivos del tratamiento y de las características del tumor.

\section{Tratamiento de la recidiva tras un tratamiento curativo}

Tras una PR, valores de PSA >0'2 ng/ml traducen una recidiva del cáncer ${ }^{45}$. Tras la radioterapia, un valor de $2 \mathrm{ng} / \mathrm{ml}$ sobre el nadir tras la radioterapia reflejan una recidiva tumoral.

Se debe hacer un esfuerzo en intentar distinguir entre la probabilidad de recidiva local exclusivamente frente a recidiva a distancia asociada o no a fallo local. El informe patológico inicial, el tiempo que ha transcurrido desde el tratamiento primario y la velocidad con que se eleva el PSA pueden ayudar en esta distinción (Tabla 6). Un tumor pobremente diferenciado, recidiva de PSA precoz, y un PSA que se eleva rápidamente orientan hacia una recidiva a distancia (enfermedad sistémica), mientras que se puede asumir un fallo local exclusivo en los pacientes con 
Tabla 4. Guías para el seguimiento tras tratamiento con intención curativa

1. En pacientes asintomáticos, se recomienda una anamnesis específica de la enfermedad, una medición del PSA sérico, acompañados de un tacto rectal como seguimiento rutinario. Se deben realizar a los 3 , 6 y 12 meses tras el tratamiento, después cada 6 meses hasta el tercer año, y posteriormente anualmente (grado B de recomendación).

2. Tras prostatectomía radical, un nivel sérico de PSA >0’2 $\mathrm{ng} / \mathrm{ml}$ se puede asociar con enfermedad residual o recidiva (grado B de recomendación).

3. Tras radioterapia, más que un valor de corte específico, la elevación del PSA 2’0 ng/ml por encima del valor nadir, es el signo más fiable de enfermedad persistente o recidiva (grado B de recomendación).

4. Tanto la palpación de un nódulo como la elevación del PSA sérico pueden ser signos de recidiva local de la enfermedad (grado B de recomendación).

5. Se recomienda la detección de una recidiva local mediante ecografía transrectal y biopsia sólo si ésta va a afectar al tipo de tratamiento (rescate, radioterapia, o cirugía). En la mayoría de los casos, la ecografía transrectal con biopsia no es necesaria previo a un tratamiento de segunda línea (grado B de recomendación).

6. Se pueden detectar las metástasis mediante TAC/RM pélvica o gammagrafía ósea. Se pueden omitir estas pruebas en pacientes asintomáticos con PSA sérico $<20 \mathrm{ng} / \mathrm{ml}$, pero los datos a este respecto son dispersos (grado B de recomendación).

7. No se recomienda la realización rutinaria de gammagrafía óseas u otras pruebas de imagen en pacientes asintomáticos. Si un paciente tiene dolor óseo, se debería considerar realizar una gammagrafía ósea independientemente de los niveles de PSA sérico (grado B de recomendación).

Tabla 5 - Guía sobre el seguimiento tras hormonoterapia

1. Se debe evaluar a los pacientes a los 3 y 6 meses tras el inicio del tratamiento. Al menos se debe realizar una medición del PSA sérico, tacto rectal y una cuidadosa evaluación de los síntomas para poder valorar la respuesta al tratamiento y los efectos secundarios.

2. Se debe ajustar el seguimiento a cada paciente en particular, de acuerdo con los síntomas, factores pronósticos y el tratamiento prescrito (grado $\mathrm{C}$ de recomendación).

3. En aquellos pacientes con enfermedad estadío MO con una buena respuesta al tratamiento, se debe realizar una revisión cada 6 meses, que debe incluir al menos una anamnesis dirigida de la enfermedad, tacto rectal y la determinación de un PSA sérico (grado C de recomendación).

4. En aquellos pacientes con enfermedad estadio M1 con una buena respuesta al tratamiento, se deben programar las revisiones cada 3-6 meses. Un seguimiento mínimo debería incluir una anamnesis dirigida de la enfermedad, tacto rectal y una medición del PSA sérico, frecuentemente complementada con la determinación de la hemoglobina, creatinina sérica, y fosfatasa alcalina (grado $\mathrm{C}$ de recomendación).

5. Cuando acontece una progresión de la enfermedad o si el paciente no responde al tratamiento prescrito, se debe individualizar el seguimiento (grado $\mathrm{C}$ de recomendación).

6. No se recomienda las pruebas de imagen de rutina en pacientes estables (grado B de recomendación).

tumores moderadamente diferenciados, recidiva tardía de PSA y PSA-DT largo (>10-12 meses). El tratamiento puede entonces orientarse según el sitio de recidiva sospechado, la situación general del paciente y las preferencias personales (Tablas 7 y 8).

La determinación del sitio de recidiva mediante pruebas de imagen tales como la gammagrafía ósea o el TAC no son de utilidad diagnóstica a menos que el PSA sérico sea $>20 \mathrm{ng} / \mathrm{ml}$ o la velocidad de PSA sea $>2 \mathrm{ng} / \mathrm{ml} /$ año. La bobina endorrectal es una técnica útil para la detección de las recidivas locales tras una PR si el PSA sérico es $>2 \mathrm{ng} / \mathrm{ml}$.

Se ha usado con éxito el PET ("positron emission tomography") con ${ }^{11} \mathrm{C}$-colina para la detección precoz de recidivas locales o a distancia de diversas neoplasias humanas. Sin embargo, sólo hay unos pocos datos publicados acerca de la eficacia clínica del PET en el CaP, por lo que no se pueden hacer conclusiones finales. Obviamente, el PET/CT no está indicado como una prueba de imagen rutinaria en la situación clínica de elevación del PSA tras tratamiento con intención curativa.

\section{Tratamiento de la recidiva tras terapia hormonal}

Los pacientes que presentan una recidiva tras el tratamiento hormonal están habitualmente en estadios más avanzados de la enfermedad y generalmente tendrán sintomas tras un periodo de tiempo relativamente corto tras el comienzo de la elevación 
Tabla 6. Parámetros clínicos e histopatológicos importantes predictivos de la recidiva local y sistémica tras una prostatectomía radical

\begin{tabular}{lcc}
\hline Parámetro & $\begin{array}{c}\text { Recidiva } \\
\text { local }\end{array}$ & $\begin{array}{c}\text { Recidiva } \\
\text { a distancia }\end{array}$ \\
\hline $\begin{array}{l}\text { Intervalo a la recidiva PSA } \\
\quad \leq 1 \text { año }\end{array}$ & $7 \%$ & $93 \%$ \\
1-2 años & $10 \%$ & $90 \%$ \\
$\quad>2$ años & $61 \%$ & $39 \%$ \\
$>3$ años & $74 \%$ & $26 \%$ \\
Tiempo doblaje PSA & 11,7 meses & 4,3 meses \\
Grado Gleason & & \\
2-4 & $0 \%$ & $0 \%$ \\
5-6 & $55 \%$ & $45 \%$ \\
7 & $39 \%$ & $61 \%$ \\
8-10 & $11 \%$ & $89 \%$ \\
Estadío patológico & & \\
Organoconfinado (spT2b) & $40 \%$ & $60 \%$ \\
PT3a, R0 & $54 \%$ & $46 \%$ \\
PT3a, R1 & $48 \%$ & $52 \%$ \\
PT3b & $16 \%$ & $84 \%$ \\
PTxpN1 & $7 \%$ & $93 \%$ \\
\hline
\end{tabular}

del PSA. En la mayoría de los casos la decisión de tratar o no tratar se realiza basándose en la situación de cada paciente, lo que limita el papel de las guías. Sin embargo, hay algunos algoritmos terapéuticos disponibles que se han realizado basándose en datos de ensayos clínicos prospectivos. Las recomendaciones acerca del manejo de los pacientes con fallo de la terapia hormonal se resumen en la Tabla 9.

La definición precisa de recurrencia o recidiva del CaP permanece controvertida. Recientemente, varios grupos han publicado unas recomendaciones prácticas que debieran ser seguidas cuando definamos el CaP hormono refractario ${ }^{47}$. Se debe diferenciar el CaP andrógeno independiente pero hormono-sensible del CaP verdaderamente hormono-refractario desde el inicio. El primer grupo todavía responde a maniobras hormonales secundarias, tales como la retirada del antiandrógeno, estrógenos y corticoides, mientras que el último es resistente a cualquier manipulación hormonal. La Tabla 10 enumera los criterios clave para definir un CaP hormono-refractario

Tabla 7. Opciones terapéuticas de segunda línea tras tratamiento local con intención curativa.

Manejo de la recidiva bioquímica tras prostatectomía radical

1. El tratamiento de la recidiva local mediante radioterapia de rescate con 64-66 Gy tiene mejores resultados con PSA sérico $\leq 1,5 \mathrm{ng} / \mathrm{ml}$ (grado B de recomendación).

2. Una opción es la observación en pacientes con presunta recidiva local y no aptos o no deseosos de recibir radioterapia (grado B de recomendación).

3. El mejor tratamiento de la elevación del PSA sugestivo de recidiva sistémica es la supresión androgénica precoz, ya que disminuye la frecuencia de metástasis clínicas (grado B de recomendación).

4. Cuando hay indicación de hormonoterapia se pueden usar tanto análogos de la hormona liberadora de la hormona luteinizante/orquiectomía, como bicalutamida a dosis de $150 \mathrm{mg} /$ día. (grado A de recomendación).

Manejo de la recidiva bioquímica tras radioterapia

1. La recidiva local se puede tratar mediante prostatectomía radical de rescate en pacientes cuidadosamente escogidos con problable enfermedad organoconfinada (grado C de recomendación).

2. La crioablación prostática o la braquiterapia intersticial son alternativas experimentales en pacientes no adecuados para la cirugía (grado $\mathrm{C}$ de recomendación).

3. La supresión androgénica es una opción en aquellos pacientes con problable enfermedad sistémica (grado B de recomendación).

Tabla 8. Guía para terapias de segunda línea tras tratamiento curativo

1. Probable fallo local tras prostatectomía radical: Los pacientes con supuesto fallo local pueden ser candidatos radioterapia de rescate. Se debe radiar al menos con 64 Gy y preferiblemente antes de que el PSA sea mayor de 1'5 ng/ml. Otros pacientes pueden beneficiarse más de un periodo de observación con un posible tratamiento hormonal posterior (grado B de recomendación).

2. Probable fallo local tras radioterapia: Pacientes seleccionados pueden ser candidatos a prostatectomía radical de rescate. Otros enfermos pueden beneficiarse más de un periodo de observación con un posible tratamiento hormonal posterior (grado $\mathrm{C}$ de recomendación).

3. Probable recidiva a distancia \pm local: Hay algunos datos de que el tratamiento hormonal precoz, comparado con el tratamiento tardío, puede ser beneficioso al retrasar la progresión y posiblemente consiguiendo una mejoría en la supervivencia. Los resultados son controvertidos. El tratamiento local no se recomienda salvo por razones paliativas (grado B de recomendación). 
Tabla 9. Guía para maniobras hormonales secundarias, quimioterapia y manejo paliativo en pacientes con cáncer de próstata hormono-refractario

Manipulaciones hormonales

1. Se debe mantener niveles de castración de testosterona también en los pacientes hormono-refractarios (grado C de recomendación).

2. La administración de todos los antiandrógenos debe cesar una ver que se documente la progresión del PSA (grado B de recomendación).

3. Se debe apreciar el eventual efecto de retirada del antiandrógeno tras suspender la flutamida o la bicalutamida, a las 4 y 6 semanas respectivamente (grado B de recomendación).

4. No se puede realizar una clara recomendación acerca de cuál es la medicación más efectiva en las maniobras hormonales secundarias, ya que no hay datos disponibles de ensayos clínicos aleatorizados (grado C de recomendación).

Quimioterapia

1. En pacientes con sólo elevación del PSA, es preciso documentar dos elevaciones consecutivas del PSA sérico por encima de un valor de referencia (grado $\mathrm{B}$ de recomendación).

2. Previo al tratamiento, el PSA sérico debe ser $>5 \mathrm{ng} / \mathrm{ml}$ para asegurar una correcta interpretación de la eficacia terapéutica (grado $\mathrm{B}$ de recomendación).

3. Se debe discutir con cada paciente los beneficios potenciales de la quimioterapia y los efectos secundarios esperables (grado $\mathrm{C}$ de recomendación).

4. En pacientes con CaP metastásico hormono-refractario, el docetaxel a dosis de $75 \mathrm{mg} / \mathrm{m}^{2}$ cada $3 \mathrm{semanas}$ ofrece un beneficio significativo en la supervivencia y representa el tratamiento de referencia (grado A de recomendación).

5. En pacientes con metástasis óseas sintomáticas secundarias a un CaP hormono-refractario, el tratamiento de elección es el docetaxel con prednisona (grado A de recomendación).

Tratamiento paliativo

1. Se debe ofrecer el zoledronato a pacientes con metástasis esqueléticas para prevenir complicaciones óseas (grado A de recomendación).

2. Se debe considerar de forma precoz en el manejo de las metástasis óseas dolorosas, los tratamientos paliativos tales como radionucleodos, radioterapia externa y uso adecuado de analgésicos (grado B de recomendación).

Tabla 10. Definición de cáncer de próstata hormonorefractario

1. Niveles séricos de castración de la testosterona

2. Tres elevaciones consecutivas de PSA separadas 2 semanas entre sí con dos incrementos del 50\% sobre el nadir.

3. Retirada del antiandrógeno al menos durante 4 semanas*.

4. Progresión del PSA a pesar de las maniobras hormonales secundarias*

5. Progresión de lesiones óseas o de partes blandas.

* Se debe haber realizado al menos la retirada del antiandrógeno $\mathrm{u}$ otra manipulación hormonal secundaria para poder cumplir los criterios de $\mathrm{CaP}$ hormono-refractario

\subsection{Manipulaciones hormonales secundarias}

Están disponibles múltiples opciones terapéuticas para aquellos pacientes con progresión de la enfermedad tras la supresión androgénica. Éstas incluyen la retirada del antiandrógeno, la adición de un antiandrógeno, compuestos estrogénicos, agentes adrenolíticos, y los nuevos enfoques ${ }^{48}$. El algoritmo terapéutico mostrado en la Figura 1 resume las diferentes modalidades de tratamiento y la respuesta esperada.

\subsection{Tratamiento no hormonal (quimioterapia)}

Basándose en los ensayos clínicos aleatorizados fase III, el docetaxel en combinación con la prednisona es el régimen de elección citotóxico en hombres con CaP hormono-refractario, ofreciendo un beneficio de 3 meses y una mejora significativa del dolor y de la calidad de vida comparada con la mitoxantrona ${ }^{49,50}$. El efecto beneficioso del docetaxel es independiente de la edad, dolor, del estado general al inicio o de la presencia enfermedad metastásica sintomática o asintomática. A pesar de estos resultados alentadores, el momento en el que se debe iniciar el tratamiento citotóxico en pacientes con CaP hormono-refractario sigue estando en debate, y de momento, no está indicada la quimioterapia en pacientes solamente con elevación del PSA. La indicación más adecuada de la quimioterapia es la situación clínica de paciente asintomático pero con enfermedad metastásica extensa o metástasis sintomáticas.

\subsection{Opciones terapéuticas paliativas}

Muchos pacientes con CaP hormono-refractario tienen metástasis óseas dolorosas que no son con- 


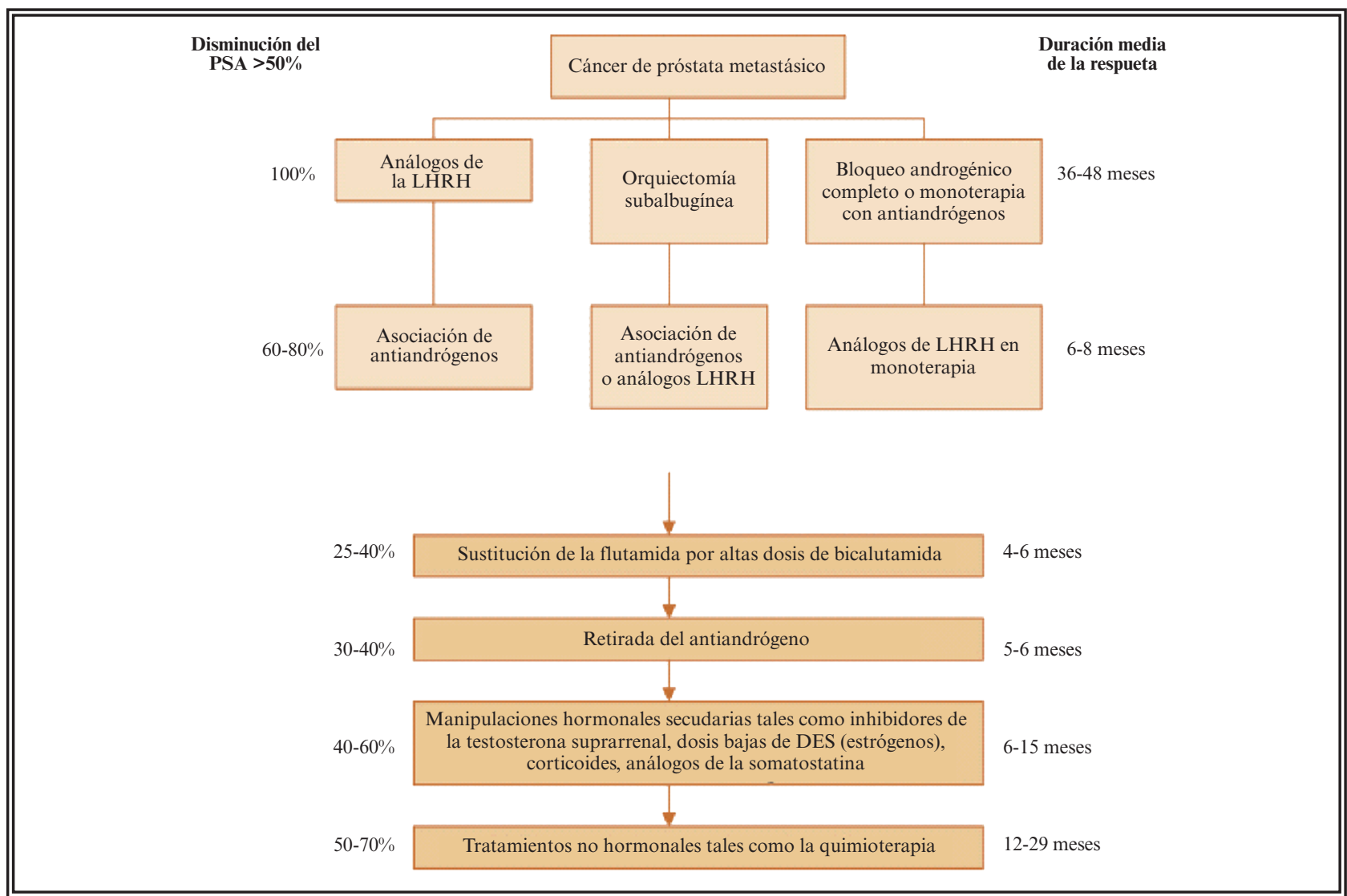

FIGURA 1. Diagrama de las opciones terapéuticas potenciales tras la progresión del PSA después de un tratamiento inicial hormonal.

troladas con la quimioterapia, por lo que los tratamientos paliativos efectivos son necesarias (por ejemplo: radioterapia externa paliativa, corticoides, analgésicos y antieméticos). El CaP hormono-refractario es habitualmente una enfermedad debilitante que afecta a varones añosos. Es necesario un enfoque multidisciplinar en el que intervengan los oncólogos médicos, los oncólogos radioterapeutas, urólogos, enfermería y los trabajadores sociales. Se debe tener en cuenta, mientras se planean los tratamientos sistémicos adicionales, cuestiones críticas del tratamiento paliativo que frecuentemente se presentan tales como el control del dolor, estreñimiento, anorexia, nausea, fatiga y depresión.

Las complicaciones más frecuentes de las metástasis esqueléticas incluyen el dolor óseo, el aplastamiento vertebral o fracturas patológicas deformantes, y la compresión medular. El uso de zoledronato ha demostrado un efecto clínicamente significativo en los pacientes con CaP hormonorefractario en términos de prevención de complicaciones esqueléticas y en la reducción del dolor, o incluso alivio completo del dolor ${ }^{51}$. Los pacientes con CaP hormono-refractario con metástasis óseas experimentan una disminución significativa de los eventos relacionados con el esqueleto, una reducción significativa de la frecuencia de fracturas patológicas, y un significativo aumento del tiempo hasta el primer evento relacionado con el esqueleto, mejorando de forma significativa, por tanto, la calidad de vida.

\section{Resumen}

El texto presente representa un resumen de la guía para el CaP de la EAU; para información más detallada y una lista completa de referencias, referimos a los lectores a la versión de texto completo. Estas guías de la EAU (ISBN 90-70244-27-6) están disponibles en la página web de la Asociación Europea de Urología (http://www.uroweb.org).

\section{Conflictos de intereses}

Dr. Axel Heindenreich tiene una relación financiera como conferenciante, consejero y Miembro de la Junta de Consejeros de Sanofi-Aventis, Novartis, Hoffmann-La Roche, Centocor. 
Durante el congreso de la EAU en Berlín 2007, el Prof. Gunnar Aus tomó parte en el simposio relacionado con el HIFU (High Intensity Focused Ultrasound) para el tratamiento del cáncer de próstata. Recibió una compensación económica de EDAP, Lyon, Francia, por presidir este simposio.

No hay cuestiones económicas que deban ser reflejados para el Prof. M. Bolla, Dr. Steven Joniau, Prof. V.B. Matveev, Prof. Hans Peter Schmid, y Prof. Filliberto Zattoni.

\section{Observaciones}

Al realizar la traducción se han identificado algunas diferencias entre la guía resumida publicada en "European Urology" y la guía completa de la EAU. Dichas discrepancias, posiblemente secundarias al proceso de maquetación del artículo original, se han comunicado a la EAU la cual nos ha autorizado a corregirlas en esta traducción.

Se exponen a continuación los cambios producidos para aclarar las posibles dudas que se pudiesen generar al comparar el artículo original en inglés y la traducción al castellano.

- Tabla 6: el "tiempo de doblaje de PSA" está dentro del apartado "intervalo hasta recidiva de PSA", cuando en la guía original consta como otro apartado diferente.

- Tabla 3: en la versión resumida, en el apartado de "localmente avanzado MO" hay dos niveles de evidencia: lb y 4 . El nivel de evidencia 4 corresponde en la guía original al apartado "localmente avanzado sintomático".

- Tabla 8: no se distingue, en la versión resumida, si el fallo local es tras radioterapia o tras prostatectomía radical quedando las recomendaciones posteriores algo confusas, lo que sí hace la guía original.

- Tabla 8: en la versión resumida, en algunas partes del texto se han intercambiado las palabras "prostatectomía" y "radioterapia" respecto a la guía completa. La traducción se ha realizado basándose en el texto de la guía original de la EAU.

- Figura 1: en la versión resumida pone "PSA >50\%" cuando en la guía original lo que dice es "disminución del PSA >50\%".

- Figura 1: como $2^{a}$ opción tras la orquiectomía subalbugínea pone en la versión resumida "añadir antiandrógenos" mientras en la guía original pone "añadir antiandrógenos o análogos LHRH".

\section{REFERENCIAS}

1. Aus G, Abbou CC, Bolla M, et al. EAU guidelines on prostate cancer. Eur Urol 2005;48:546-451.

2. US Department of Health and Human Services, Public Health Service, Agency for Health Care Policy and Research 1992, p. 115-27. http://www.ahcpr.gov/.

3. Bray F, Sankila R, Ferlay J, Parkin DM. Estimates of cancer incidence and mortality in Europe in 1995. Eur $J$ Cancer 2002;38:99-166.
4. Black RJ, Bray F, Ferlay J, Parkin DM. Cancer incidence and mortality in the European Union: cancer registry data and estimates of national incidence for 1990. Eur J Cancer 1997;33: 1075-1107.

5. Gronberg H, Damber L, Damber JE. Familial prostate cancer in Sweden: a nationwide register cohort study. Cancer 1996;77: 138-143.

6. Carter BS, Beaty TH, Steinberg GD, Childs B, Walsh PC. Mendelian inheritance of familial prostate cancer. Proc Natl Acad Sci USA 1992;89:3367-3371.

7. Sobin LH, Wittekind C, editors. TNM classification of malignant tumours. ed. 6 New York: Wiley-Liss; 2002.

8. Gleason DF, Mellinger GT. Prediction of prognosis for prostatic adenocarcinoma by combined histological grading and clinical staging. J Urol 1974;111:58-64.

9. De Koning HJ, Liem MK, Baan CA, Boer R, Schroder FH, Alexander FE. Prostate cancer mortality reduction by screening: power and time frame with complete enrolment in the European Randomized Screening for Prostate Cancer (ERSPC) trial. Int J Cancer 2002;98:268-273.

10. Schmid H-P, Riesen W, Prikler L. Update on screening for prostate cancer with prostate specific antigen. Crit Rev Oncol Hematol 2004;50:71-78.

11. Smith RA, Cokkinides V, Eyre HJ. Cancer screening in the United States 2007: a review of current guidelines, practices, and prospects. CA Cancer J Clin 2007;57:90-104.

12. Aus G, Becker C, Franze'n S, Lilja H, Lodding P, Hugosson J.Cumulative prostate cancer risk assessment with the aid of the free-to-total prostate specific antigen ratio. Eur Urol 2004; 45:160-165.

13. Thompson IM, Pauler DK, Goodman PJ, et al. Prevalence of prostate cancer among men with a prostate-specific antigen level $\leq 4.0$ ng per milliliter. N Engl J Med 2004;350:2239-2246.

14. Sun L, Moul JW, Hotaling JM, et al. Prostate-specific antigen (PSA) and PSA velocity for prostate cancer detection in men aged $<50$ years. BJU Int 2007;99:753-757.

15. Guichard G, Larre' S, Gallina A, et al. Extended 21-sample needle biopsy protocol for diagnosis of prostate cancer in 1000 consecutive patients. Eur Urol 2007;52:430-435.

16. Morote J, Lopez M, Encabo G, de Torres I. Value of routine transition zone biopsies in patients undergoing ultrasound-guided sextant biopsies for the first time. Eur Urol 1999;35: 294-297.

17. Djavan B, Ravery V, Zlotta A, et al. Prospective evaluation of prostate cancer detected on biopsies 1,2, 3 and 4: when should we stop? J Urol 2001;166:1679-1683.

18. Chodak GW, Thisted RA, Gerber GS, Johansson JE, Adolfsson J, Jones GW, Chisholm GD, Moskovitz B, Livne PM, Warner J. Results of conservativemanagement of clinically localized prostate cancer. N Engl J Med 1994;330:242-248.

19. Albertsen PC, Hanley JA, Gleason DF, Barry MJ. Competing risk analysis of men aged 55 to 74 years at diagnosis managed conservatively for clinically localized prostate cancer. JAMA 1998;280:975-980.

20. Klotz L. Active surveillance for prostate cancer: for whom? J Clin Oncol 2005;23:8165-8169.

21. Zhang L, Loblaw A, Klotz L. Modeling prostate specific antigen kinetics in patients on active surveillance. J Urol 2006;176: 1392-1397.

22. Bill-Axelson A, Holmberg L, Ruutu M, et al. Scandinavian Prostate Cancer Group study no. 4: radical prostatectomy versus watchful waiting in early prostate cancer. N Eng J Med 2005;352:1977-1984.

23. Walsh PC. The discovery of the cavernous nerves and development of nerve sparing radical retropubic prostatectomy. J Urol 2007; $177: 1632-1635$. 
24. Heidenreich A, Ohlmann $\mathrm{CH}$, Polyakov S. Anatomical extent of pelvic lymphadenectomy in patients undergoing radical prostatectomy. Eur Urol 2007;52:29-37.

25. Joniau S, Hsu C-Y, Lerut E, et al. A pretreatment table for the prediction of final histopathology after radical prostatectomy in clinical unilateral T3a prostate cancer. Eur Urol 2007;51:388396.

26. Ward JF, Slezak JM, Blute ML, Bergstralh EJ, Zincke H. Radical prostatectomy for clinically advanced (cT3) prostate cancer since the advent of prostate-specific antigen testing: 15year outcome. BJU Int 2005;95:751-756.

27. Kumar S, Shelley M, Harrison C, Coles B, Wilt TJ, Mason MD. Neo-adjuvant and adjuvant hormone therapy for localised and locally advanced prostate cancer. Cochrane Database Syst Rev 2006;CD006019.

28. McLeod DG, Iversen P, See WA, Morris T, Armstrong J, Wirth MP, Casodex Early Prostate Cancer Trialists'Group. Bicalutamide $150 \mathrm{mg}$ plus standard care vs standard care alone for early prostate cancer. BJU Int 2006;97:247-254.

29. Messing EM, Manola J, Yao J, et al. Eastern Cooperative Oncology Group study EST 3886: immediate versus deferred androgen deprivation treatment in patients with node-positive prostate cancer after radical prostatectomy and pelvic lymphadenectomy. Lancet Oncol 2006;7:472-479.

30. Kupelian P, Kuban D, Thames H, et al. Improved biochemical relapse-free survival with increased external radiation doses in patients with localized prostate cancer: the combined experience of nine institutions in patients treated in 1994 and 1995. Int J Radiat Oncol Biol Phys 2005;61:415-419.

31. Zelefsky MJ, Leibel SA, Gaudin PB, et al. Dose escalation with three-dimensional conformal radiation therapy affects the outcome in prostate cancer. Int J Radiat Oncol Biol Phys 1998;41: 491-500.

32. Pollack A, Zagars GK, Smith LG, et al. Preliminary results of a randomized radiotherapy dose-escalation study comparing 70 Gy with 78 Gy for prostate cancer. J Clin Oncol 2000;18:39043911.

33. Bolla M, Collette L, Blank L, et al. Long-term results with immediate androgen suppression and external irradiation in patients with locally advanced prostate cancer (an EORTC study): a phase III randomised trial. Lancet 2002;360:103-106.

34. Bolla M, Gonzalez D, Warde P, et al. Improved survival in patients with locally advanced prostate cancer treated with radiotherapy and goserelin. N Engl J Med 1997;337:295-300.

35. Ash D, Flynn A, Batterman J, de Reijke T, Lavagnini P, Blank L, ESTRA/EAU Urological Brachytherapy Group, EORTC Radiotherapy Group. ESTRO/EAU/EORTC recommendations on permanent seed implantation for localized prostate cancer. Radiother Oncol 2000;57:315-321.

36. Machtens S, Baumann R, Hagemann J, et al. Long-term results of interstitial brachytherapy (LDR-brachytherapy) in the treatment of patients with prostate cancer. World $\mathrm{J}$ Urol 2006;24:289-295.

37. Bottke D, Wiegel T. Adjuvant radiotherapy after radical prostatectomy: indications, results and outcome. Urol Int 2007;78: 193-197.

38. Aus G. Current status of HIFU and cryotherapy in prostate cancer - a review. Eur Urol 2006;50:927-934.

39. Seidenfeld J, Samson DJ, Hasselblad V, et al. Single-therapy androgen suppression inmenwith advanced prostatecancer: a systematic review and meta-analysis. Ann Intern Med 2000; 132:566-577.

40. Samson DJ, Seidenfeld J, Schmitt B, et al. Systematic review and meta-analysis of monotherapy compared with combined androgen blockade for patients with advanced prostate carcinoma. Cancer 2002;95:361-376.

41. Iversen P, Tyrell CJ, Kaisary AV, et al. Casodex (bicalutamide) 150-mg monotherapy compared with castration in patients with previously untreated nonmetastatic prostate cancer: results from two multicenter randomized trials at a median follow-up of 4 years. Urology 1998;51:389-396.

42. Loblaw DA, Virgo KS, Nam R, et al. Initial hormonal management of androgen-sensitive metastatic, recurrent, or progressive prostate cancer: 2006 update of an American Society of Clinical Oncology practice guideline. J Clin Oncol. 2007;25: 1596-1605.

43. Hussain M, Tangen CM, Higano C, et al. Absolute prostatespecific antigen value after androgen deprivation is a strong independent predictor of survival in new metastatic prostate cancer-data from Southwest Oncology Group Trial 9346 (INT0162). J Clin Oncol 2006;24:3984-3990.

44. Moul JW,Wu H, Sun L, et al. Early versus delayed hormonal therapy for prostate specific antigen only recurrence of prostate cancer after radical prostatectomy. J Urol 2004;171:11411147.

45. Cookson MS, Aus G, Burnett AL, et al. Variation in the definition of biochemical recurrence in patients treated for localized prostate cancer: the American Urological Association Prostate Guidelines for localized prostate cancer update panel report and recommendations for a standard in the reporting of surgical outcomes. J Urol 2007;177:540-545.

46. Roach III M, Hanks G, Thames Jr H, et al. Defining biochemical failure following radiotherapy with or without hormonal therapy in men with clinically localized prostate cancer: recommendations of the RTOG-ASTRO Phoenix Consensus Conference. Int J Radiat Oncol Biol Phys 2006;65:965-974.

47. Bubley GJ, Carducci M, Dahut W, et al. Eligibility and response guidelines for phase II clinical trials in androgen-independent prostate cancer: recommendations from the Prostate-Specific Antigen Working Group. J Clin Oncol 1999;17:3461-3467.

48. Ryan CJ, Small EJ. Role of secondary hormonal therapy in the management of recurrent disease. Urology 2003;62(Suppl 1):87-94.

49. Petrylak DP, Tangen CM, Hussain MH, et al. Docetaxel and estramustine compared with mitoxantrone and prednisone for advanced refractory prostate cancer. N Engl J Med 2004;351: 1513-1520.

50. Tannock IF, de Wit R, Berry WR, et al. TAX 327 Investigators. Docetaxel plus prednisone or mitoxantrone plus prednisone for advanced prostate cancer. N Engl J Med 2004;351:1502-1512.

51. Saad F, Gleason DM, Murray R, et al. A randomized, placebocontrolled trial of zoledronic acid in patients with hormonerefractory metastatic prostate carcinoma. J Natl Cancer Inst 2002;94:1458-1468.

Autor para correspondencia: Dr. Axel Heidenreich Department of Urology, Division of Oncological Urology, University of Köln, Kerpener Strasse 62, 50924 Köln, Germany Tel.: +49 221478 86577; Fax: +49 22147887901

E-mail: axel.heidenreich@uk-koeln.de

Correspondencia autor traducción: Dr. Antonio Rosino Sánchez Servicio de Urología. Hospital Morales Meseguer.

Marqués de los Vélez - s/n. 30008 Murcia

Tel: +34968360900.

E-mail: a_rosino@yahoo.es

Información artículo: Revisión - Cáncer de próstata 